\title{
Validation of two nonlinear system identification techniques using an experimental testbed
}

\author{
V. Lenaerts ${ }^{\mathrm{a}}$, G. Kerschen ${ }^{\mathrm{a}, *}$, J.-C. Golinval $^{\mathrm{a}}$, M. Ruzzene ${ }^{\mathrm{b}}$ and E. Giorcelli ${ }^{\mathrm{c}}$ \\ ${ }^{a}$ Vibrations et Identification des Structures, University of Liège, Chemin des Chevreuils 1 (B52), B-4000 Liège, \\ Belgium \\ ${ }^{\mathrm{b}}$ School of Aerospace Engineering, Georgia Institute of Technology, 270 Ferst Dr., Atlanta, GA 30332-0150, USA \\ ${ }^{\mathrm{c} D i p a r t i m e n t o}$ di Meccanica, Politecnico di Torino, C.so Duca Abruzzi 24, 10129, Torino, Italy
}

\begin{abstract}
A Tribute to Professor Bruno Piombo
C'est dans le cadre d'une mission scientifique de l'action européenne COST F3 à Politecnico di Torino que j'ai rencontré le toujours souriant et blagueur Bruno Piombo. C’est avec émotion que je me remémorerai nos quelques rencontres. Vincent Lenaerts

Questo lavoro e' dedicato al caro amico Bruno, la cui prematura scomparsa ha lasciato un vuoto incolmabile. I suoi insegnamenti mi aiutano quotidianamente, e la sua amicizia mi manca moltissimo. Massimo Ruzzene
\end{abstract}

\begin{abstract}
The identification of a nonlinear system is performed using experimental data and two different techniques, i.e. a method based on the Wavelet transform and the Restoring Force Surface method. Both techniques exploit the system free response and result in the estimation of linear and nonlinear physical parameters.
\end{abstract}

Keywords: Nonlinear system identification, wavelet transform, coulomb friction, restoring force surface method

\section{Introduction}

During the last ten years, we have witnessed quite significant progress in the field of nonlinear system identification. In the early nineties, the study of the single degree of freedom (SDOF) Duffing oscillator was more or less ubiquitous in the literature. Recently, techniques which enables us to deal with systems of increasing complexity have been introduced (see for instance [1,7,13]. For more details about nonlinear system identification, the interested reader is referred to the book of Worden and Tomlinson published in 2001.

The present study results from a collaboration of the University of Liege and Politecnico di Torino within the framework of the European COST Action F3. It was carried out in Torino in August 1999 with the objective to compare two different identification techniques, namely a method based on the Wavelet transform (WT) and the Restoring Force Surface (RFS) method. The WT method is based on the detection of the decay envelope which is fitted to its theoretical formulation using nonlinear averaging techniques. The method can be applied to identify both damping and stiffness non-linearities. In the experiment presented in this work, however, stiffness nonlinearities are not significant, and the identification is therefore limited to the damping mechanism coefficients. The RFS technique expresses the restoring force as a mathematical function of the displacement and of the velocity using least squares fitting. The comparison was performed using the free response of an experimental system with nonlinear damping. It is emphasised that its behaviour could be modelled using a SDOF system. 


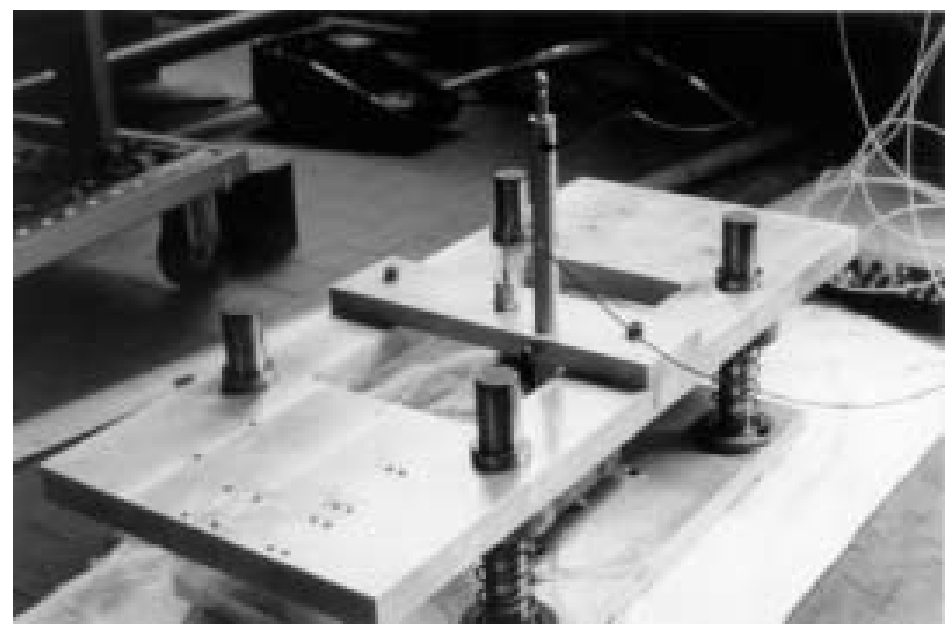

Fig. 1. Experimental set-up.
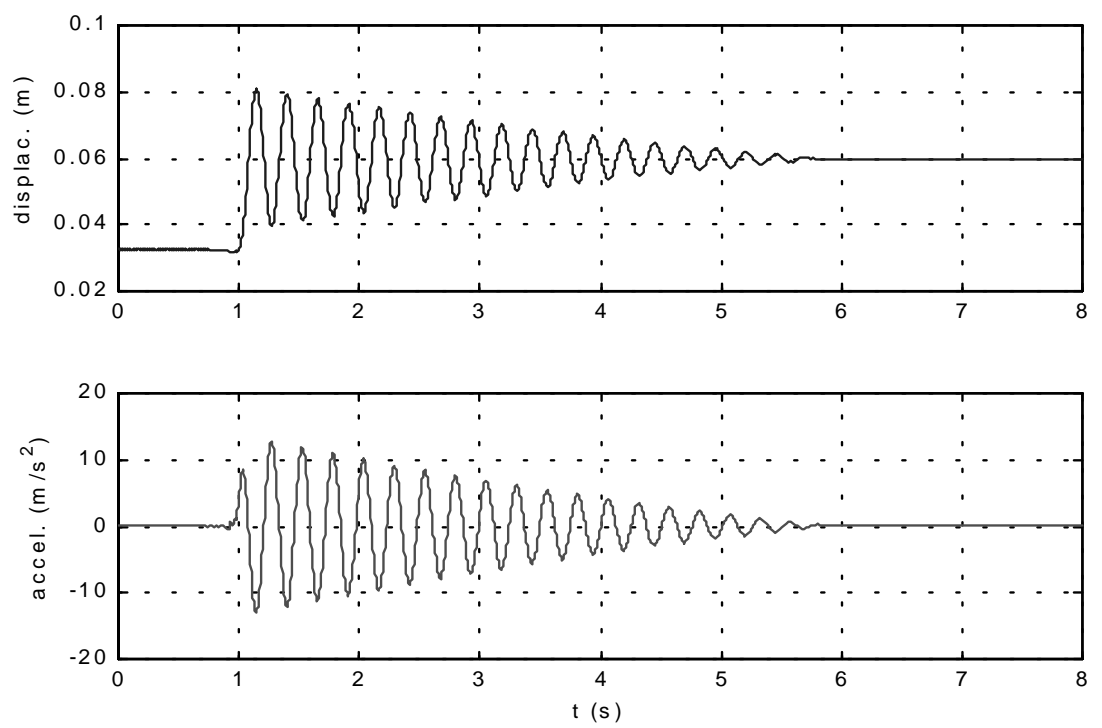

Fig. 2. Example of recorded displacement and acceleration.

\section{The wavelet transform for nonlinear system identification}

In this work an attempt is made to extend the WT analysis to the free vibration of nonlinear SDOF systems. The WT may be considered as an improved tool for envelope and phase estimation [8] with respect to the Hilbert Transform (HT) which has been extensively used for the identification of nonlinear systems [4,5].

A very general formulation for the damping force acting on the system is considered here, the dissipation mechanism being modelled as a n-th order power series of the velocity. The analytical expression of the free response of systems with this kind of damping has been derived using first order approximation methods, i.e. the Method of Multiple Scales and the Method of Averaging [6]. The presence of nonlinear stiffness can also be considered : the analytical derivation for the free response has been derived through the methods mentioned above for the case of stiffness of the generalised odd order $\mathrm{m}$. 


\subsection{Response of a nonlinear single degree of freedom system}

The equation of motion of a weakly nonlinear SDOF system can be written with the well known expression:

$$
\ddot{u}(t)+\omega_{0}^{2} \cdot u(t)=\varepsilon \cdot f(u, \dot{u})
$$

where $\omega_{0}=\sqrt{k / m}$ is the natural angular frequency of the linear system, $\varepsilon$ is a small dimensionless parameter and $f(u, \dot{u})$ a general nonlinear function of $u, \dot{u}$. With the assumption of $\varepsilon$ being small, a number of approximate methods can be used for the determination of the analytical solution of Eq. (1) [6]. In particular, using the method of averaging [6], a solution can be sought in the form:

$$
u(t)=k(t) \cdot \cos \left(\omega_{0} \cdot t+\gamma(t)\right)=k(t) \cdot \cos \phi(t)
$$

where $k(t)$ and $\gamma(t)$ are the amplitude and the phase modulation of the system free response respectively.

For small $\varepsilon$, it can be stated that the variations of $k(t)$ and $\gamma(t)$ over one period of $\cos \phi$ are negligible when compared to the variations of $\phi(t)$. By averaging over one period, the expressions describing the slow variations of $k(t)$ and $\gamma(t)$ can be obtained:

$$
\begin{aligned}
& \dot{k}=-\frac{\varepsilon}{2 \pi \cdot \omega_{0}} \cdot \int_{0}^{2 \pi} \sin \phi \cdot f\left(k \cdot \cos \phi,-\omega_{0} \cdot k \cdot \sin \phi\right) d \phi \\
& \dot{\gamma}=-\frac{\varepsilon}{2 \pi \cdot k \cdot \omega_{0}} \cos \int_{0}^{2 \pi} \cos \phi \cdot f\left(k \cdot \cos \phi,-\omega_{0} \cdot k \cdot \sin \phi\right) d \phi
\end{aligned}
$$

Equations (3) and the Method of Averaging in general allow to obtain an approximate analytical expression describing the free behaviour of a SDOF system, for different forms of the function $f(u, \dot{u})$.

This work focuses on the study of systems with combined generalised nonlinear damping mechanisms, for which the function $f(u, \dot{u})$ has the following form:

$$
\varepsilon \cdot f(u, \dot{u})=\varepsilon \cdot f(\dot{u})=-\sum_{i=0}^{n} \mu_{i} \cdot|\dot{u}|^{i} \cdot \operatorname{sign}(\dot{u})
$$

where $n$ is the maximum order considered in the damping mechanism and $\mu_{1}$ is the i-th damping coefficient, normalised to the mass of the system.

Equation (4) allows to consider the simultaneous effect of different simple damping mechanisms acting on the system, i.e. Coulomb damping, viscous (linear) damping, aerodynamic forces (quadratic damping), etc. In particular Eq. (4) gives the opportunity to approximate with a polynomial function the restoring force given by a real dissipation mechanism.

An approximate expression of the free response of a SDOF system with a combined damping mechanism of the generalised order $\mathrm{n}$ can still be found from Eq. (3) obtaining:

$$
\dot{k}=-\sum_{i=0}^{n} \frac{\varepsilon}{\sqrt{\pi}} \cdot \mu_{i} \cdot \omega_{0}^{i-1} \cdot \frac{\Gamma(i / 2+1)}{\Gamma(i / 2+3 / 2)} \cdot k^{i}=-\sum_{i=0}^{n} c_{i} \cdot k^{i}
$$

and

$$
\dot{\gamma}=\frac{\varepsilon}{\pi \cdot \omega_{0} \cdot k} \cdot \int_{0}^{\pi} \cos \phi \cdot \sum_{i=0}^{n} \mu_{i} \cdot \omega_{0}^{i} \cdot k^{i}(\sin \phi)^{i} \cdot d \phi=0
$$

Equation (5) is a first order differential equation, whose analytical solution for a generalised value of $n$ is trivial. In this work, the theoretical envelope of a system with combined damping has been determined by numerical integration of Eq. (5). 


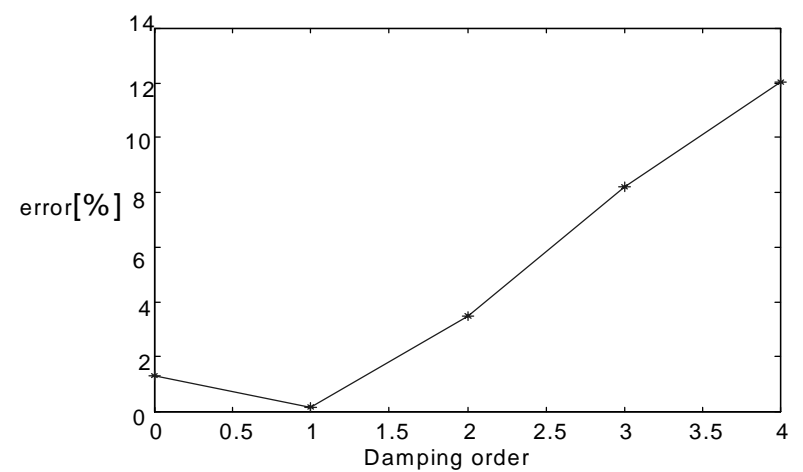

WT (a)

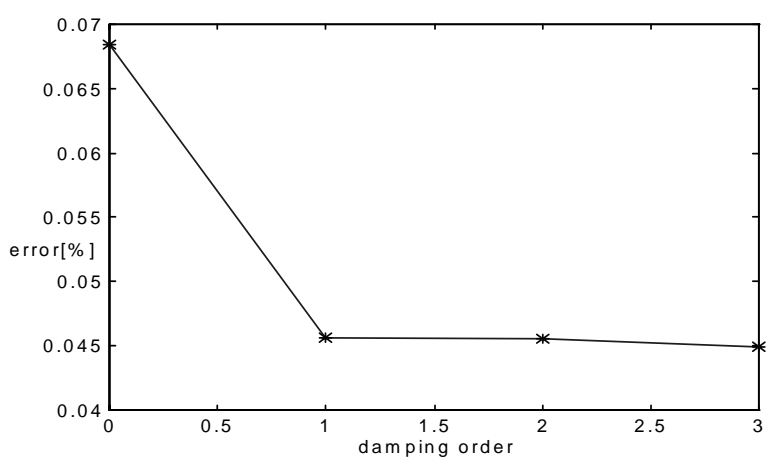

RFS (b)

Fig. 3. Evolution of the MSE with the damping order.

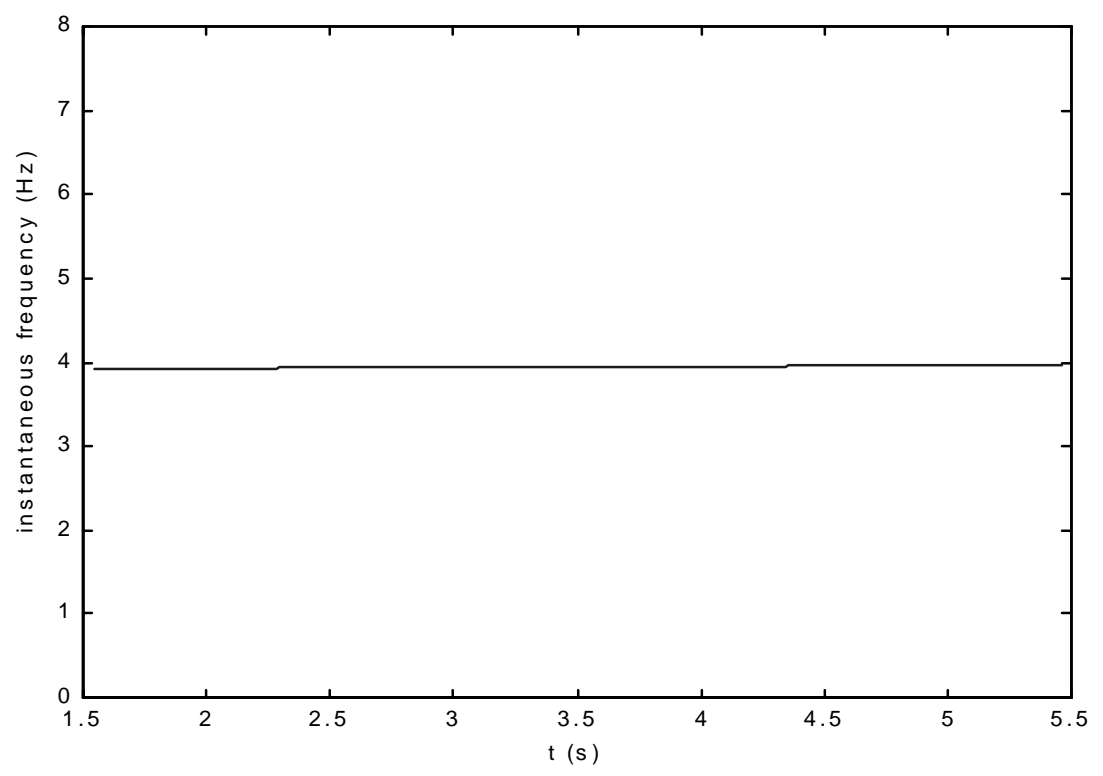

Fig. 4. Instantaneous frequency of a recorded signal.

\subsection{Identification of the envelope and instantaneous frequency of a signal}

The WT of a signal $x(t)$ is an example of a time-scale decomposition obtained by dilating and translating along the time axis a chosen analysing function named 'wavelet'. The continuous WT is defined as follows:

$$
W_{g}(a, b)=\frac{1}{\sqrt{a}} \cdot \int_{-\infty}^{+\infty} x(t) \cdot g^{*}\left(\frac{t-b}{a}\right) d t
$$

where $b$ is the parameter localising the wavelet function in the time domain, $a$ is the dilation parameter defining the analysing window stretching and $g^{*}$ is the complex conjugate of the basic wavelet function. The wavelet function used in this work is the Morlet's wavelet. It can be shown [9] how the WT gives a time-frequency representation of the signal performing a linear transformation.

The WT has already been used for modal parameters identification in reference [8]. In particular, it has been highlighted how the WT of signals expressed by Eq. (2) is:

$$
W_{g}(a, b)=\sqrt{a} \cdot k(t) \cdot e^{-\left(a \cdot \phi(t)-\omega_{w}\right)^{2}} \cdot e^{j \phi(t)}
$$




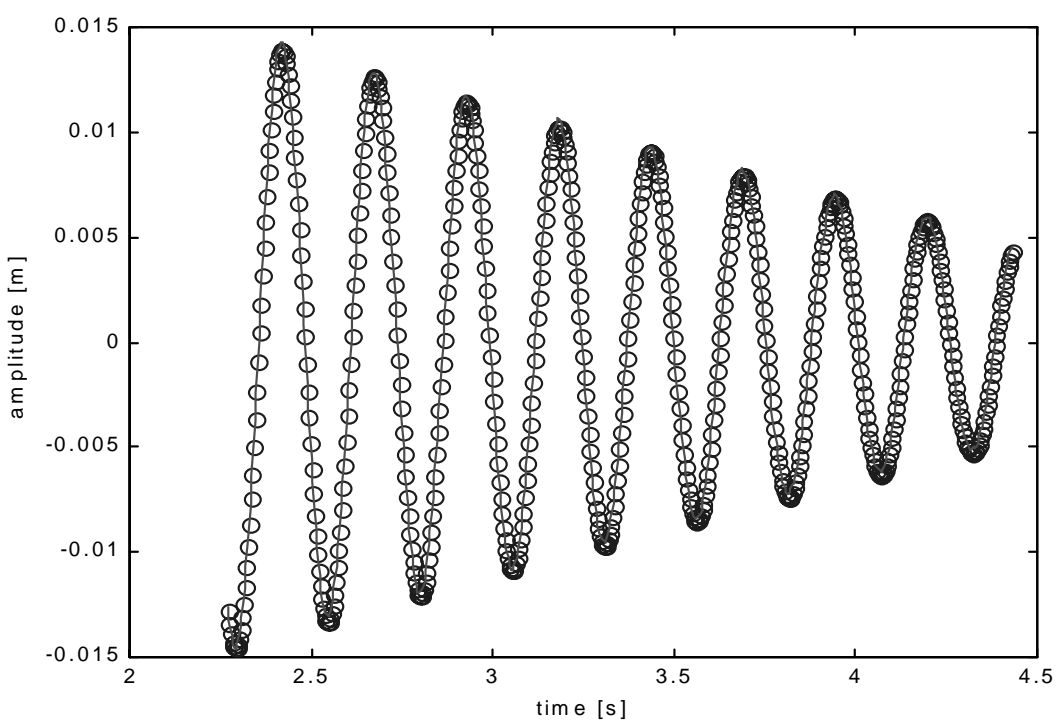

(a) Displacement

Restoring force

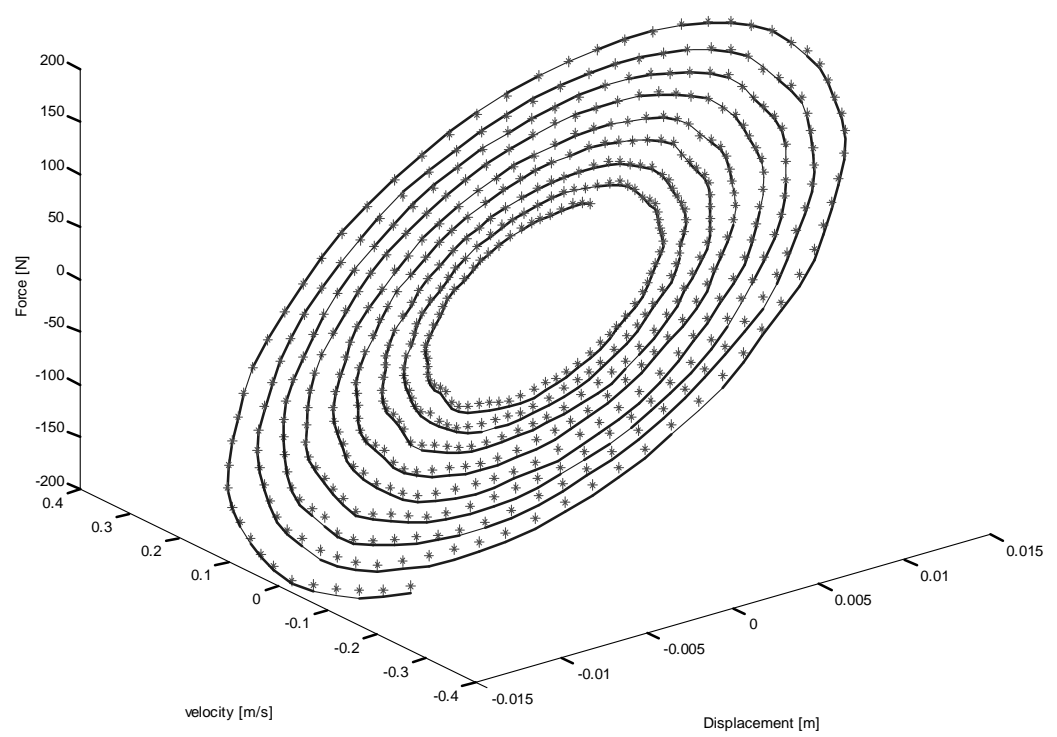

(b) Restoring force

Fig. 5. Comparison between measured (-) and predicted (o) signals (WT method).

where $k(t)$ and $\phi(t)$ are generally varying in time. For a fixed frequency value, the modulus of Eq. (8) is directly proportional to the envelope of the signal $k(t)$ while the time derivative of the phase of $W_{g}(a, b)$ gives the instantaneous frequency content of the signal. 


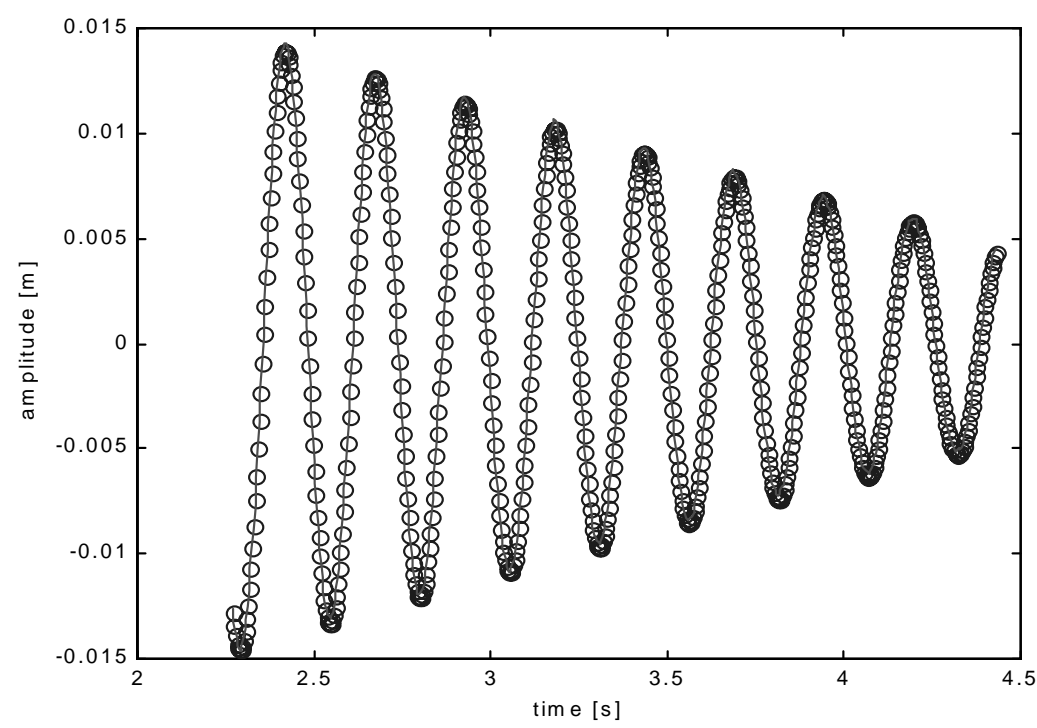

(a) Displacement

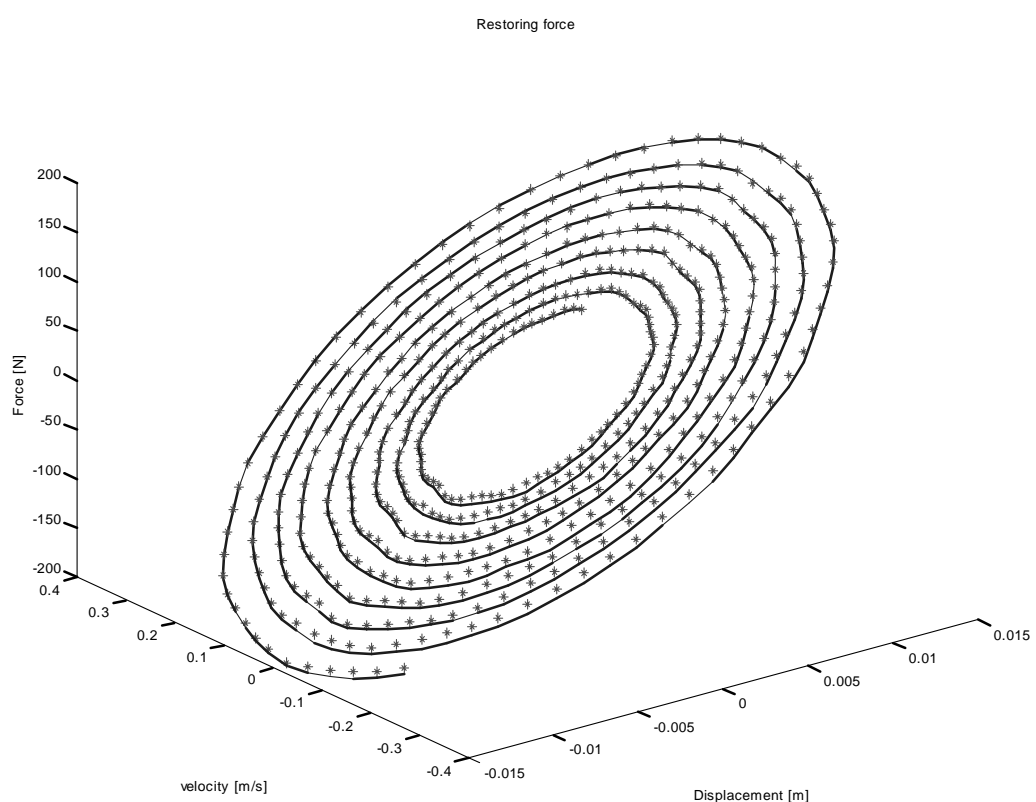

(b) Restoring force

Fig. 6. Comparison between measured (-) and predicted (o) signals (RFS method).

\subsection{Estimation of the damping coefficients: Least square solution}

The theoretical solution for a combined damping mechanism defined by Eq. (4), together with the estimation procedure based on the WT has been used for the identification of the damping coefficients $\mu_{i}$ in Eq. (4). Equation (5) gives the amplitude of the free response of a system with a known damping mechanism, i.e. with known coefficients $\mu_{i}(i=0, \ldots, n)$. 
If an identification procedure has to be performed, the damping coefficients $\mu_{i}$ obviously represent the unknowns of the problem, while the amplitude of the decay can be rather easily estimated using the WT (Eq. (8)). If $k(t)$ in Eq. (8) is differentiated with respect to time $t$ and if expression Eq. (5) is used, an equation in the $n$ unknowns $c_{i}$, directly proportional to $\mu_{i}$ is obtained:

$$
\dot{k}(t)-\sum_{i=0}^{n} c_{i} \cdot k^{i}(t)=0
$$

where $k(t)$ is the envelope estimated by the WT.

To reduce the influence of noise present in the estimation of $k(t)$, it is preferable to integrate Eq. (9) with respect to time:

$$
\int_{t_{0}}^{t_{m}} \dot{k} \cdot d \tau-\sum_{i=0}^{n}\left(c_{i} \int_{t_{0}}^{t_{m}} k^{i} \cdot d \tau\right)=0
$$

where $t_{m}-t_{0}$ represents the time duration of the analysed signal.

Let us write for the sake of simplicity:

$$
x(t)=\int_{t_{0}}^{t_{m}} \dot{k}(\tau) \cdot d \tau=k(t)-k\left(t_{0}\right) ; \quad y_{i}(t)=\int_{t_{0}}^{t_{m}} k^{i}(\tau) \cdot d \tau
$$

the $n$ coefficients $c_{i}$ can be easily obtained in closed form by imposing the stationarity of the following error function:

$$
e=\sum_{j=1}^{m}\left[x\left(t_{j}\right)-\sum_{i=0}^{n} c_{i} \cdot y_{i}\left(t_{j}\right)\right]^{2}=0
$$

Hence a linear system is obtained with $n$ equations in the $n$ unknowns $c_{i}$. Once the $n$ coefficients $c_{i}$ have been found, the damping coefficients $\mu_{i}$ can be easily calculated from Eq. (5).

\section{Restoring force surface method}

The RFS method has been widely used in the past $[2,3,10,11]$ mainly because of its simplicity and efficiency.

It is based on Newton's second law:

$$
m \ddot{u}(t)+f(u, \dot{u})=x(t)
$$

where $m$ is the mass of the system, $x(t)$ is the external force and $f(u, \dot{u})$ is the internal displacement and velocity dependent force often referred to as restoring force. If the external force and the acceleration are sampled simultaneously at regular intervals, the value of the restoring force at each sampling instant can be computed from the following relationship:

$$
f\left(t_{i}\right)=x\left(t_{i}\right)-m \ddot{u}\left(t_{i}\right)
$$

where $t_{i}$ represents the ith sampled value of the signals. If the displacements and the velocities, obtained by integrating the measured acceleration data, are estimated at each sampling instant, a triplet of values $u\left(t_{i}\right), \dot{u}\left(t_{i}\right), f\left(t_{i}\right)$ can be obtained. Each triplet specifies a point in the phase plane $(u, \dot{u})$ and the corresponding amplitude of the restoring force. Using this data, a continuous representation of the surface can be constructed which gives an easily understandable representation of the system nonlinearity. A mathematical model may be obtained by fitting the data triplets:

$$
\sum_{r}^{n_{1}} \mu_{r} \cdot \dot{u}_{i}^{r}+\sum_{j}^{n_{2}} \chi_{j} \cdot u_{i}^{j}=x_{i} / m-\ddot{u}_{i} \quad(i=1, \ldots, p)
$$

This equation can be assembled into a matrix by considering all the $p$ recorded samples: 
Table 1

Identified parameters of the low dissipation system

\begin{tabular}{cccccccc}
\hline Case $^{\circ}$ & Initial displacement $\mathrm{cm}$ & $\mu_{0}(\mathrm{WT})$ & $\mu_{0}(\mathrm{RFS})$ & $\mu_{1}(\mathrm{WT}) \mathrm{N} / \mathrm{m}$ & $\mu_{1}(\mathrm{RFS}) \mathrm{N} / \mathrm{m}$ & $K(\mathrm{WT}) \mathrm{Ns} / \mathrm{m}$ & $K(\mathrm{RFS}) \mathrm{Ns} / \mathrm{m}$ \\
\hline 1. & -2.5 & 0.13 & 0.12 & 0.28 & 0.27 & 13104 \\
1. & -2 & 0.12 & 0.12 & 0.28 & 0.27 & 13118 \\
2. & -1.5 & 0.12 & 0.12 & 0.26 & 0.24 & 13121 \\
3. & -0.5 & 0.11 & 0.11 & 0.29 & 0.25 & 13184 \\
4. & 2 & 0.12 & 0.12 & 0.28 & 0.29 & 13113 \\
5. & 2.5 & 0.14 & 0.14 & 0.28 & 0.30 & 131 \\
6. & not measured & 0.13 & 0.13 & 0.29 & 0.30 & 13126 \\
7. & not measured & 0.14 & 0.13 & 0.27 & 0.30 & 13107 \\
8. & not measured & 0.14 & 0.13 & 0.26 & 0.26 & 13090 \\
\hline
\end{tabular}

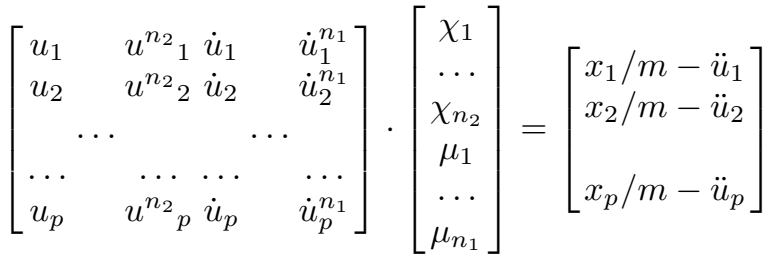

which may be written in the much simpler form:

$$
\mathbf{A c}=\mathbf{b}
$$

The vector $c$ of unknown parameters may be determined using the singular value decomposition (SVD).

The normalised mean-square error (MSE) which may be regarded as a measure of the accuracy of the identification is defined as:

$$
M S E(f)=\frac{100}{N \cdot \sigma_{f}^{2}} \sum_{i=1}^{N}\left(f_{i}-f_{i}^{\prime}\right)^{2}
$$

where $f_{i}$ and $f_{i}^{\prime}$ contain the measured and predicted values of the restoring force respectively.

In practice, the orders of the polynomials $n_{1}$ and $n_{2}$ used in Eqs (15) and (16) are not known before hand. In order to have an indication about which terms should be included in the model and which terms can be safely discarded, the significance factor can be used. Each term of the model $\vartheta(t)$, for example $\vartheta(t)=\chi_{3} u^{3}$, may be used independently to generate a time series which will have variance $\sigma_{\vartheta}^{2}$. The significance factor is then defined as:

$$
s_{\vartheta}=100 \cdot \frac{\sigma_{\vartheta}^{2}}{\sigma_{\text {tot }}^{2}}
$$

where $\sigma_{\text {tot }}^{2}$ is the variance of the term $x / m-\ddot{u}$, including all the model terms. At this point, it should be emphasized that the RFS method has a very appealing way of determining the functional form of the nonlinearity since the restoring force can be plotted in a three-dimensional graph against the displacement and velocity. Roughly speaking, $s_{\vartheta}$ represents the percentage of contribution to the model variance of the term $\vartheta(t)$ of the restoring force. After the estimation of the parameters, the significance factors are computed and all the terms contributing less than a specified threshold value may be discarded.

This method may be applied using different types of excitation (random, sinusoidal, sweep sine, etc.). In the present study, the RFS method is applied to the free response of the system, i.e. by setting $x(t)=0$ in the equations. In this particular case, the application of the method requires the previous estimation of the system mass as all the coefficients in Eqs (15) and (16) are mass normalised.

It is emphasised that the knowledge of the mass is also required for the method based on the WT.

\section{Experimental validation of the two nonlinear system identification techniques}

The two aforementioned techniques have been applied to the measured free response of a mass suspended by four springs in a way that its dynamic behaviour can be easily assumed to be that of a SDOF system. 
Table 2

Comparison of the results

\begin{tabular}{lcccccc}
\hline & $K \mathrm{~N} / \mathrm{m}$ & $\sigma_{K} \mathrm{~N} / \mathrm{m}$ & $\mu_{0}$ & $\sigma_{\mu_{0}}$ & $\mu_{1} \mathrm{Ns} / \mathrm{m}$ & $\sigma_{\mu_{1}} \mathrm{Ns} / \mathrm{m}$ \\
\hline WT & 13115.3 & 28 & 0.13 & 0.01 & 0.277 & 0.01 \\
RFS & 13114.6 & 40 & 0.12 & 0.01 & 0.276 & 0.02 \\
\hline
\end{tabular}

\subsection{Experimental set-up}

The WT technique only requires the measurement of the displacement of the system whereas the RFS method needs to know displacement, velocity and acceleration. Considering that velocities and displacements may be obtained, with some care, by integration of the acceleration responses, the RFS method requires to measure the accelerations of the system. Note that this procedure leads to good results, provided the low frequency components, always present in the measured and integrated signals, are previously filtered out. In order to compare the results obtained with the two methods, displacements and accelerations were measured.

The experimental system is composed of a rectangular plate suspended over a massive base assuring the stability of the system (see Fig. 1). The plate is linked to the base by four springs and is constrained to move in the vertical direction by four linear supports, hence reproducing, as closely as possible, the behaviour of a spring-mass SDOF system.

The suspended mass including the sensors and the mounting is equal to $21.36 \mathrm{~kg}$ and the global stiffness of the system in the vertical direction is evaluated to $12625 \mathrm{~N} / \mathrm{m}$ from a static test. The mass is coupled with the linear guides by four linear bushes with circulating balls, with the aim of reducing the friction between the moving parts. This type of dissipation is not the only one that can be expected in the system, since also structural damping is likely to be present. The decay response of the system may be presumed to be caused by a combination of a friction damping term, and of an exponentially decaying term associated with linear dissipations. The simultaneous presence of these effects makes the system nonlinear. The aim of the identification procedure is to verify this presumption and to detect other possible forms of dissipation mechanism and/or nonlinearity.

\subsection{Test description}

After imposing an initial displacement to the mass, the free decay responses were obtained by release of the system. Different initial displacement values were first considered, so that the estimation procedures could be tested in different conditions, namely with the system undergoing different numbers of oscillations before returning at rest. Another set of time histories was recorded by imposing an initial velocity to the mass. The estimation procedures were carried out for all the test configurations in order to check the repetitiveness and the stability of the identification methods.

The data were sampled at $256 \mathrm{~Hz}$ for an acquisition time of 8 seconds, corresponding to 2048 samples, that is about the time required for the system to return at rest. An example of measured displacement and acceleration data is presented in Fig. 2.

\subsection{Parameter identification}

The evolution of the MSE with the damping order is presented in Fig. 3 for both methods. In the case of the WT method, the minimum of the function identifies the order of the damping mechanisms, which here is estimated equal to 1. For the RFS method, the MSE in Fig. 3(b) first decreases rapidly and then very slowly when the correct damping order is reached. For all test configurations, a first order damping mechanism, i.e. including Coulomb friction and linear damping, has thus been estimated. The significance factor has also confirmed these findings. The presumptions briefly discussed in Section 4.1 were therefore meaningful. The corresponding identified parameters are listed in Table 1.

The behaviour of the springs within the range of displacements considered in the tests, has been observed to be approximately linear. This is confirmed by the fact that the instantaneous frequency of all the signals remains approximately constant for the duration of the responses. An example of instantaneous frequency is presented in Fig. 4. In the case of the WT method, the value of the linear stiffness K has been evaluated by calculating the mean 
value of the instantaneous frequency and by using the estimated value of the suspended mass given in Section 4.1 $\left(k=m \cdot \omega_{0}^{2}\right)$. The instantaneous frequency is calculated through numerical differentiation of the phase of the WT, according to procedures outlined in Ruzzene et al. In both methods, the resulting value of the dynamic stiffness $k$ is slightly different than the one estimated from the static test.

The comparison between the original and the reconstructed signals using the WT method is given in Fig. 5, while Fig. 6 shows the comparison with the signal identified through the restoring force method. Figures 5(a) and 6(a) compare the measured and reconstructed decays, while Figs 5(b) and 6(b) show the measured term and the restoring force reconstructed from the identified parameters, that is according to the following expression:

$$
f(u, \dot{u})=m \cdot \mu_{0} \cdot \operatorname{sign}(\dot{u})+m \cdot \mu_{1} \cdot \dot{u}+K \cdot u
$$

It can be observed that the measured and predicted signals agree to the point where the difference between them is hardly visible.

Finally, the results obtained by the WT and RFS methods are compared in Table 2. Note that this table gives the average values and the corresponding standard deviations for all the results obtained in the different test configurations.

\section{Conclusions}

The purpose of this study was to identify the behaviour of an experimental system exhibiting nonlinear damping. This system could be modelled using a SDOF model. A good match could be found between the parameters obtained by two rather different techniques: a well established one (the RFS method), and an original technique (the WT method).

The WT technique has the advantage that only the displacement signals are to be measured whereas the RFS method needs the knowledge of the acceleration, velocity and displacement signals. For this reason, a lot of care must be dedicated in assuring and checking a perfect simultaneity between the signals. In most practical cases, it is a hard task to perform, since the sensors and particularly their amplification devices often introduce very small time lags between recorded responses. In our case, a small phase difference was experienced and hence, in a first stage, the results obtained by the RFS method did not match the results obtained by the WT technique. It was thus decided to calculate the displacement data by double integration of the acceleration signal.

A drawback of the WT technique is that it always requires a theoretical model as a baseline for the identification. The RFS method has however a very appealing way of determining the functional form of the nonlinearity since the restoring force can be plotted in a three-dimensional graph against the displacement and velocity. An even better representation of the restoring force can be obtained by taking a cross section of this three-dimensional plot along the axes where either the displacement or the velocity is equal to zero.

Although techniques which can deal with more complex systems are now at our disposal, we believe that, due to their appealing features, there is still room for these two methods.

\section{Acknowledgements}

The author G. Kerschen is supported by a grant from the Belgian National Fund for Scientific Research (FNRS) which is gratefully acknowledged.

\section{References}

[1] D. Adams and R.J. Allemang, A frequency domain method for estimating the parameters of a non-linear structural dynamic model trhough feedback. Mechanical Systems and Signal Processing 14 (2000), 637-656.

[2] P. Atkins and K. Worden, Proceedings of the 15th IMAC, Orlando, Florida, Identification of a multi-degree-of-freedom nonlinear system 1997, pp. 1023-1028.

[3] S.W.R. Duym, J.F.M. Schoukens and P.A.N. Guillaume, A local restoring force surface method, Modal Analysis 11(3) (1996), 117-132. 
[4] M. Feldman, Proceedings of the 15th IMAC, Orlando, Florida, Vibration analysis of non-symmetric elastic force systems via the Hilbert transform, 1997, pp. 1017-1022.

[5] K.S. Mohammad and G.R. Tomlinson, Proceedings of the 7th IMAC, Las Vegas, Nevada, A simple method of accurately determining the apparent damping in non-linear structures, 1989, pp. 1336-1346.

[6] A.H. Nayfeh and D.T. Mook, Nonlinear oscillations, New York, John Wiley \& Sons, 1979.

[7] C.M. Richards and R. Singh, Identification of multi-degree-of-freedom non-linear systems under random excitations by the reverse-path spectral method. Journal of Sound and Vibration 213 (1998), 673-708.

[8] M. Ruzzene et al., Natural frequencies and damping identification using wavelet transform: application to real data. Mechanical Systems and Signal Processing 11(2) (1997), 207-218.

[9] W.J. Staszewski and G.R. Tomlinson, Application of the wavelet transform to fault detection in a spur gear. Mechanical Systems and Signal Processing 8(3) (1994), 289-307.

[10] K. Worden, Parametric and non parametric identification of nonlinearity in structural dynamics, Ph.D. Thesis, Heriot-Watt University, 1989.

[11] K. Worden, J.R. Wright, M.A. Al-Hadid and K.S. Mohammad, Experimental identification of multi degree of freedom nonlinear systems using restoring force methods. Modal Analysis 9(1) (1994), 35-55.

[12] K. Worden and G.R. Tomlinson, Nonlinearity in Structural Dynamics: Detection, Identification and Modelling, Bristol, Institute of Physics Publishing, 2001.

[13] J.R. Wright, M.F. Platten, J.E. Cooper and M. Sarmast, Proceedings of the International Conference on Structural System Identification, Kassel, Identification of multi-degree-of-freedom weakly non-linear systems using a model based in modal space, $2001, \mathrm{pp} .49-68$. 

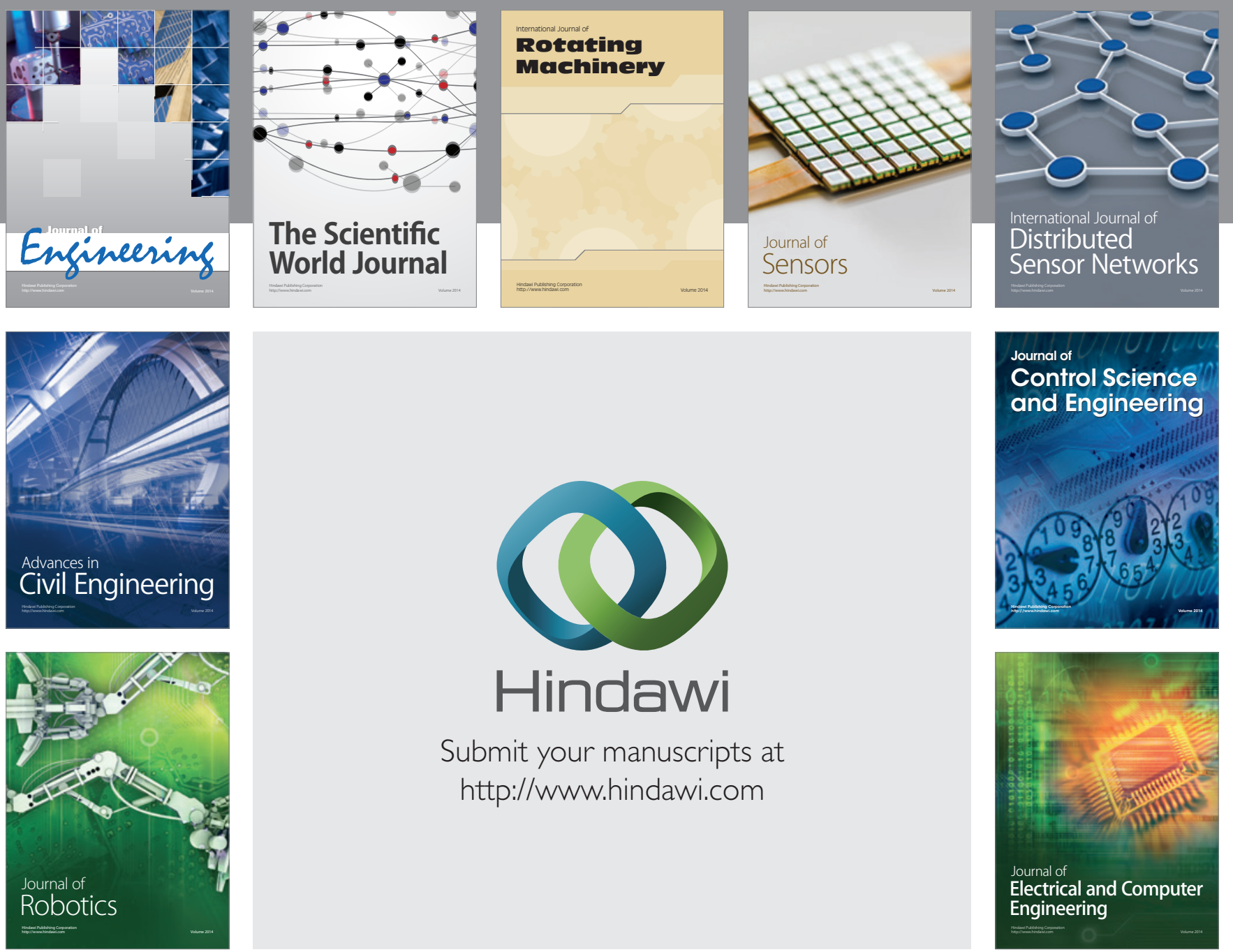

Submit your manuscripts at

http://www.hindawi.com
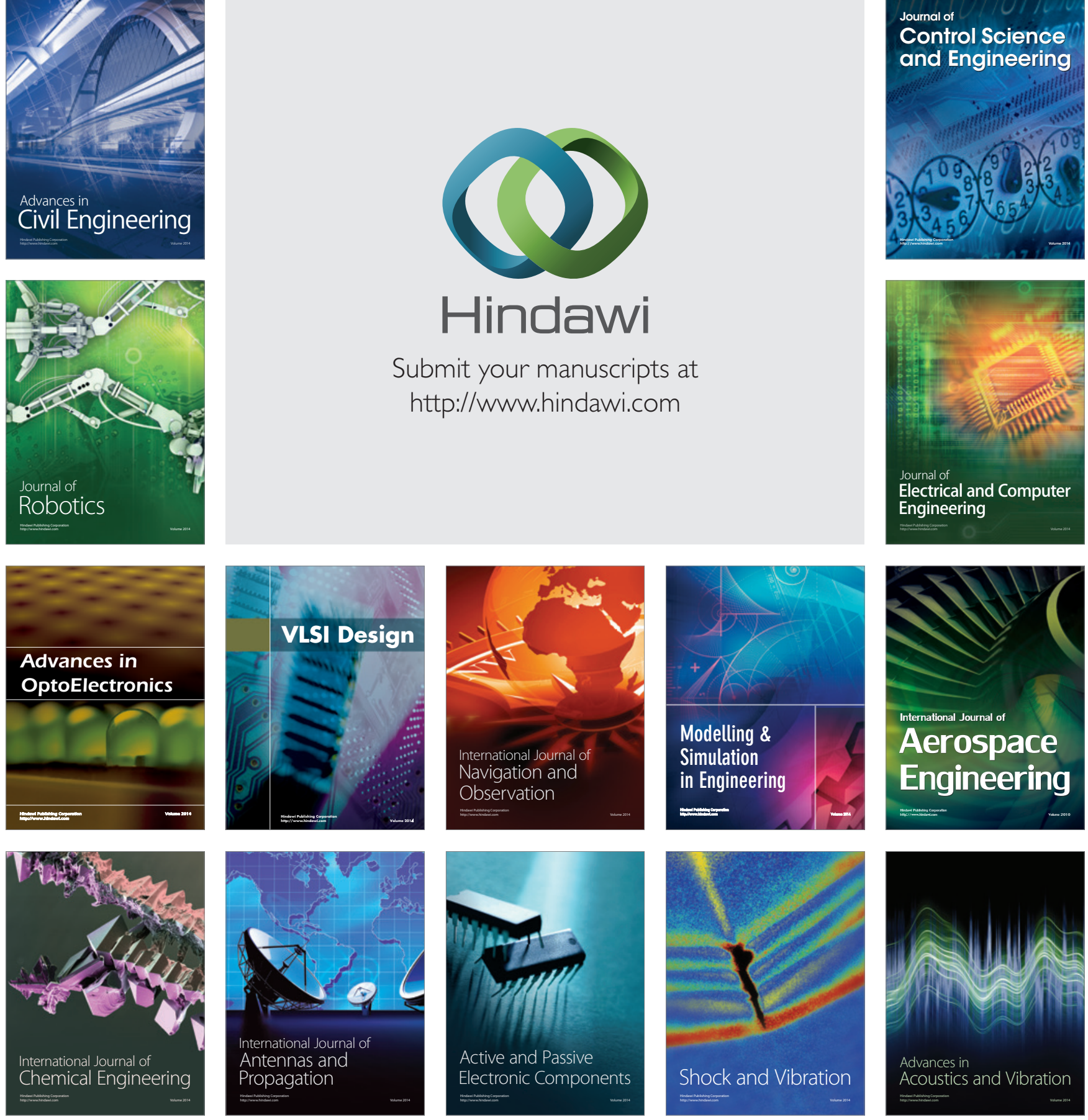